\title{
Drag force in strongly coupled, anisotropic plasma at finite chemical potential
}

\author{
Somdeb Chakraborty and Najmul Haque \\ Theory Division, Saha Institute of Nuclear Physics, \\ 1/AF Bidhannagar, Kolkata-700 064, India \\ E-mail: somdeb.chakraborty@saha.ac.in, najmul.haque@saha.ac.in
}

ABSTRACT: We employ methods of gauge/string duality to analyze the drag force on a heavy quark moving through a strongly coupled, anisotropic $\mathcal{N}=4, \mathrm{SU}(N)$ super YangMills plasma in the presence of a finite $\mathrm{U}(1)$ chemical potential. We present numerical results valid for any value of the anisotropy parameter and the U(1) charge density and arbitrary direction of the quark velocity with respect to the direction of anisotropy. In the small anisotropy limit we are also able to furnish analytical results.

KEYwords: Quark-Gluon Plasma, Gauge-gravity correspondence, AdS-CFT Correspondence, Holography and quark-gluon plasmas

ARXIV EPRINT: 1410.7040 


\section{Contents}

1 Introduction $\quad 1$

2 The dual geometry 3

3 Drag force $\quad 6$

$\begin{array}{llr}3.1 & \text { Results } & 10\end{array}$

$\begin{array}{lll}\text { 3.2 Small anisotropy and small charge limit analysis } & 15\end{array}$

$\begin{array}{lll}4 & \text { Discussion and conclusion } & 17\end{array}$

$\begin{array}{ll}\text { A Details of the numerical scheme used } & 17\end{array}$

\section{Introduction}

Recent heavy ion experiments at the Relativistic Heavy Ion Collider (RHIC) [1, 2] and the Large Hadron Collider (LHC) have provided precious insights into the rich physics underlying quark-gluon plasma (QGP), simultaneously presenting an enormous challenge to understand the wealth of experimental data from a theoretical standpoint. One of the most fascinating results to emerge out of the data obtained at RHIC is that the quark-gluon plasma formed as an outcome of the heavy ion collisions is dominated by strong coupling effects $[3,4]$. The strong coupling scenario urges for novel techniques to study such systems, since the conventional field-theoretic approach relies upon perturbative tools and, as such, may not be reliable in the strong coupling domain. The AdS/CFT correspondence or more generally, the gauge/string duality [5-8], on the other hand, seems to be tailor-made to handle such situations. The best understood and most investigated example of this duality conjectures an equivalence between type IIB string theory on $A d S_{5} \times S^{5}$ and $\mathcal{N}=4, \mathrm{SU}(N)$ super Yang-Mills (SYM) theory living on the $(3+1)$-dimensional boundary of the $A d S_{5}$ space. It enables us to extract information about the strongly coupled SYM theory by studying the weakly coupled string dual. Since then the duality has been generalized in various directions to embrace a larger variety of gauge theories under its scope.

One of the frontier areas where the duality has been applied with reasonable success is the physics of quark-gluon plasma. Collisions of heavy nuclei are usually marked by a non-zero impact parameter which results in a pressure gradient building up in the region where the two colliding nuclei overlap. The initial coordinate-space anisotropy is transformed into an observed momentum-space anisotropy, via the interactions between the produced particles finally leading to an anisotropic particle distribution. The relativistic ideal hydrodynamical models tasted early success in explaining soft collective flow and hadronic spectra at RHIC. This provided empirical evidence in favor of fast thermalization 
and isotropization - at time scales $\sim 0.5 \mathrm{fm}$. In an attempt to make better agreement with experimental results this was subsequently generalized to incorporate viscous effects, which, however, predicted the presence of a sizable pressure anisotropy. Hydrodynamical simulations suggest that the transverse pressure exceeds the longitudinal (along the beam direction) one with the disparity being the most conspicuous for time $\leq 2 \mathrm{fm}$. Hence, to describe a realistic situation, it is desirable to take into account the presence of anisotropy in the system. Though in a real plasma the strength of anisotropy is expected to die away with time, in many cases it suffices to consider the anisotropy to be constant over the time-scale of interest. Encouraged by the field-theoretical studies on anisotropic QGP [9-12], there have also been a spate of activities related to studying strongly coupled, anisotropic QGP via the gauge/string duality [13-19]. Recently, Mateos and Trancanelli [20,21] constructed a gravity solution firmly embedded in type IIB superstring theory that is dual to a topologically deformed SYM theory, where the topological deformation injects anisotropy into the theory. Although the source of anisotropy in such a model is radically different from that in actual QGP, nevertheless, one expects this model to encapsulate the telltale signatures of anisotropy on various QGP observables. Various quantities related to heavy quark observables were subsequently computed in this model [22-39]. An interesting scenario to consider is when the QGP formed is endowed with a finite baryon number density. At RHIC, the QGP is assumed to carry a finite, albeit small, baryon number density and is characterized by a high temperature. Forthcoming collider programs at the Facilty for Antiproton and Ion Research (Gesellschaft für Schwerionenforschung, Germany) and the Nuclotronbased Ion Collider fAcility (Joint Institute for Nuclear Research, Russia) are expected to thoroughly explore plasma characterized by a high baryon number density and moderate temperature. To study such systems using the AdS/CFT toolkit, one needs to introduce a $\mathrm{U}(1)$ chemical potential in the dual gravity description. The easiest way to implement this is to consider black branes carrying U(1) $R$-charge. Recently, the authors of $[40,41]$ presented a type IIB solution dual to $\mathcal{N}=4$, anisotropic SYM plasma and carrying a non-zero $\mathrm{U}(1)$ charge. We use this supergravity background to compute the drag force experienced by a heavy quark moving in an anisotropic, charged thermal medium. While the drag force in both anisotropic [23] and charged [42,43] plasma have been computed previously, it is of interest to see the outcome of the interplay between the two parameters. Motivated by this, we investigate in this paper the drag force experienced by an energetic quark moving through hot, anisotropic, charged plasma and uncover some rather remarkable results.

The paper is organized as follows. In section 2 we elaborate upon the gauge theory that we wish to consider and the dual gravity description which was first studied in [40, 41]. In section 3 we study the drag force acting on a massive quark as it moves through the plasma and present numerical results. Further, by restricting to the small anisotropy and small charge density regime we are able to furnish analytical results for the drag force. Finally, we conclude with a discussion of our results in section 4 . 


\section{The dual geometry}

In $[20,21]$ Mateos and Trancanelli obtained a type IIB supergravity solution dual to a topologically deformed, thermal $\mathcal{N}=4, \mathrm{SU}(N)$ SYM theory at large 't Hooft coupling $\lambda=g_{\mathrm{YM}}^{2} N$. The gauge theory of interest is a deformed cousin of the $\mathcal{N}=4$ SYM theory where the deformation is effected by a topological term,

$$
S=S_{\mathrm{SYM}}+\frac{1}{8 \pi^{2}} \int \theta\left(x^{3}\right) \operatorname{Tr} F \wedge F
$$

where $\left\{t, x^{1}, x^{2}, x^{3}\right\}$ define the gauge theory coordinates and the $\theta$-term, that depends upon only one of the gauge theory space coordinates (in this case $x^{3}$ ), is responsible for injecting anisotropy into the theory thereby spoiling the $\mathrm{SO}(3)$ symmetry. Now a remnant $\mathrm{SO}(2)$ symmetry is preserved only in the $x^{1}-x^{2}$ plane. While trying to relate the situation to heavy ion collisions it is then only natural to identify the $x^{3}$ direction with the beam direction. Aiming for a more realistic model, this was recently generalized to encompass the case where the plasma is characterized by a finite $\mathrm{U}(1)$ charge density [40, 41]. In the dual picture one considers a Einstein-Maxwell-dilaton-axion system in 10-dimensional type IIB supergravity compactified upon $S^{5}$. The non-linear Kaluza-Klein reduction of $10-$ dimensional supergravity on 5-dimensions results in an Abelian gauge field. The relevant 5-dimensional action is given in the Einstein frame by,

$$
S=\frac{1}{2 \kappa^{2}} \int_{\mathcal{M}} \sqrt{-g}\left(\mathcal{R}+12-\frac{1}{2}(\partial \phi)^{2}-\frac{1}{2} e^{2 \phi}(\partial \chi)^{2}-\frac{1}{4} F_{M N} F^{M N}\right)+\frac{1}{2 \kappa^{2}} \int_{\partial \mathcal{M}} \sqrt{-\gamma} 2 K
$$

where $2 \kappa^{2} \equiv 16 \pi G_{5}=\frac{8 \pi^{2}}{N^{2}} 1$ is the gravitational coupling in 5-dimensions, $F_{M N}=\partial_{[M} A_{N]}$. $\phi$ and $\chi$ are the dilatonic and the axionic excitations respectively and the last term is the usual Gibbons-Hawking surface term defined on the 4-dimensional boundary $\partial \mathcal{M}$ of the 5-dimensional manifold $\mathcal{M}$ and $\gamma_{\mu \nu}$ being the metric on $\partial \mathcal{M}$. The 5 -dimensional solution, given in the string frame, is taken to have the form,

$$
\begin{aligned}
d s^{2} & =\frac{1}{u^{2}}\left(-\mathcal{F} \mathcal{B} d t^{2}+\left(d x^{1}\right)^{2}+\left(d x^{2}\right)^{2}+\mathcal{H}\left(d x^{3}\right)^{2}+\frac{d u^{2}}{\mathcal{F}}\right) \equiv G_{M N} d X^{M} d X^{N}, \\
\chi & =a x^{3}, \quad \phi=\phi(u), \quad \mathcal{H}=e^{-\phi}
\end{aligned}
$$

where $a$ is a measure of anisotropy. The axion $\chi$ is dual to the $\theta$-term in the gauge theory. $u$ indicates the radial coordinate with the gauge theory living at $u=0 . X^{M}$, $\{M=0, \ldots, 9\}$ are the 10-dimensional coordinates. The gauge theory coordinates are defined by $t=X^{0}$ and $x^{i}=X^{i}(u=0)\{i=1,2,3\}$. The metric components $\mathcal{F}, \mathcal{B}, \mathcal{H}$ are only functions of the radial coordinate $u$. Evidently, in an isotropic theory one sets $\mathcal{H}=1$. The black brane horizon is defined by the radial coordinate $u_{H}$ such that $\mathcal{F}\left(u_{H}\right)=$ 0 . We further demand that asymptotically the metric becomes $A d S_{5}$ which imposes the constraints: $\phi(0)=0, \mathcal{F}(0)=\mathcal{B}(0)=\mathcal{H}(0)=1$. Interestingly, the authors in [41] found two classes of solution, which they termed as prolate and oblate depending upon whether

\footnotetext{
${ }^{1}$ We have set the radius $R$ of $S^{5}$, which is a constant in the Einstein frame, to be unity.
} 
$\mathcal{H}\left(u_{H}\right)>1$ or $\mathcal{H}\left(u_{H}\right)<1$. This, in turn, corresponds to whether $a$ is real or imaginary. In this paper, we shall only work with the prolate solution implying $a \in \mathbb{R}$. The temperature $T$ and the entropy density $s$ can easily be found as,

$$
\begin{aligned}
T & =-\frac{1}{4 \pi} \mathcal{F}_{H}^{\prime} \sqrt{\mathcal{B}_{H}} \\
& =\sqrt{\mathcal{B}_{H}}\left(\frac{e^{-\frac{\phi_{H}}{2}}}{16 \pi u_{H}}\left(16+a^{2} e^{7 \frac{\phi_{H}}{2}} u_{H}^{2}\right)-\frac{e^{2 \phi_{H}} Q^{2} u_{H}^{5}}{24 \pi}\right) \\
s & =\frac{N^{2} e^{-\frac{5}{4} \phi_{H}}}{2 \pi u_{H}^{3}}
\end{aligned}
$$

where $Q$ is a constant related to the $\mathrm{U}(1)$ charge density on the gauge theory side and the prime indicates derivative with respect to the radial coordinate. In the above we have introduced the notations: $\mathcal{F}\left(u_{H}\right) \equiv \mathcal{F}_{H}$ and so on. The gauge field has only one non-vanishing component,

$$
A_{t}(u)=-\int_{u_{H}}^{u} Q \sqrt{\mathcal{B}} e^{\frac{3}{4} \phi} u d u
$$

supplemented by the constraint that it vanishes on the horizon, i.e., $A_{t}\left(u_{H}\right)=0$ whence the $\mathrm{U}(1)$ chemical potential is obtained as

$$
\mu=\int_{0}^{u_{H}} Q \sqrt{\mathcal{B}} e^{\frac{3}{4} \phi} u d u
$$

Note that setting $a=0$ we recover the temperature of the RN-AdS black brane temperature,

$$
T_{a=0}=\frac{1}{2 \pi u_{H}}\left(2-q^{2}\right)
$$

where we have defined $q \equiv \frac{u_{H}^{3} Q}{2 \sqrt{3}}$. As $q \rightarrow \sqrt{2}$ we approach the extremal RN-AdS solution which sets an upper bound on $q$ as $q_{\max }^{2}=2$. It has been argued in [41] that as one switches on a non-trivial dilaton profile with $a>0$ the horizon of the anisotropic RN-AdS black brane is always greater than its isotropic cousin signifying that the extremal limit can not be accessed. The metric functions $\mathcal{F}, \mathcal{B}, \mathcal{H}$ and the dilaton profile $\phi(u)$ can be found out analytically only in the small anisotropy limit by perturbing around the $a=0$ solution. Otherwise, they can be obtained numerically for any value of $a$. In figure 1 we provide numerical plots of these functions for some sample values of the parameters. ${ }^{2}$ For future reference, let us also discuss the case when both $a$ and $q$ are kept small, whence, it is possible to furnish some analytical results. In this regime of the parameter space let us write the relevant functions as,

$$
\begin{aligned}
& \mathcal{F}(u)=\mathcal{F}_{0}(u)+a^{2}\left(\hat{\mathcal{F}}_{0}(u)+\hat{\mathcal{F}}_{2}(u) q^{2}+\mathcal{O}(q)^{4}\right)+\mathcal{O}(a)^{4} \\
& \mathcal{B}(u)=\mathcal{B}_{0}(u)+a^{2}\left(\hat{\mathcal{B}}_{0}(u)+\hat{\mathcal{B}}_{2}(u) q^{2}+\mathcal{O}(q)^{4}\right)+\mathcal{O}(a)^{4} \\
& \phi(u)=\phi_{0}(u)+a^{2}\left(\hat{\phi}_{0}(u)+\hat{\phi}_{2}(u) q^{2}+\mathcal{O}(q)^{4}\right)+\mathcal{O}(a)^{4}
\end{aligned}
$$

\footnotetext{
${ }^{2}$ See appendix A for details of the numerical scheme followed.
} 


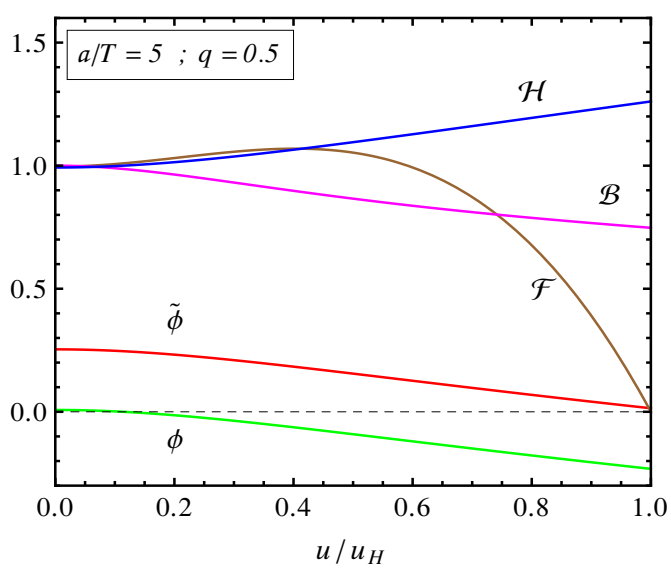

(a)

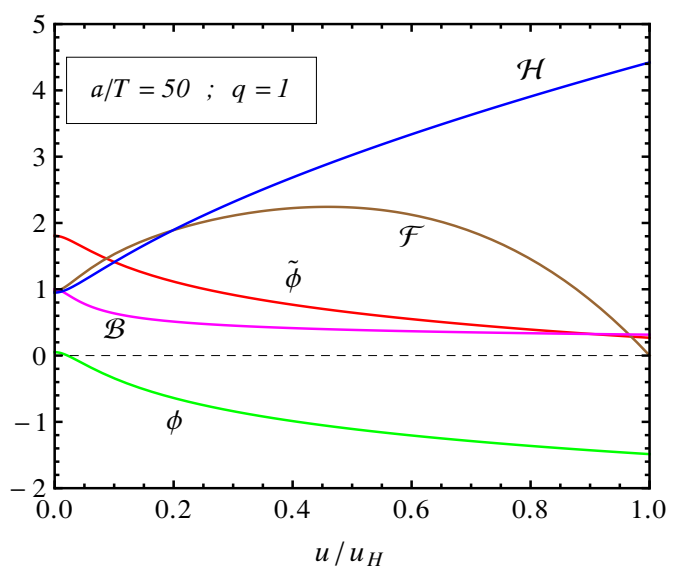

(b)

Figure 1. (a) shows numerical results for $\mathcal{F}, \mathcal{B}, \mathcal{H}, \phi$ and $\tilde{\phi}$ for $a / T=5, q=0.5$. In (b) the same quantities have been plotted with $a / T=50, q=1.0$.

where

$$
\begin{aligned}
\mathcal{F}_{0}(u)= & 1-\left(\frac{u}{u_{H}}\right)^{4}+q^{2}\left[\left(\frac{u}{u_{H}}\right)^{6}-\left(\frac{u}{u_{H}}\right)^{4}\right] \\
\hat{\mathcal{F}}_{0}(u)= & \frac{1}{24 u_{H}^{2}}\left[8 u^{2}\left(u_{H}^{2}-u^{2}\right)-10 u^{4} \log 2+\left(3 u_{H}^{4}+7 u^{4}\right) \log \left(1+\frac{u^{2}}{u_{H}^{2}}\right)\right], \\
\hat{\mathcal{F}}_{2}(u)= & \frac{1}{24 u_{H}^{4}\left(u^{2}+u_{H}^{2}\right)}\left[7 u^{8}+6 u^{2} u_{H}^{6}+u^{4} u_{H}^{4}(25 \log 2-12)+u^{6} u_{H}^{2}(25 \log 2-1)\right. \\
& \left.-\left(u^{2}+u_{H}^{2}\right)\left(12 u^{6}+7 u^{4} u_{H}^{2}+6 u_{H}^{6}\right) \log \left(1+\frac{u^{2}}{u_{H}^{2}}\right)\right], \\
\mathcal{B}_{0}(u)= & 1 \\
\hat{\mathcal{B}}_{0}(u)= & -\frac{u_{H}^{2}}{24}\left[\frac{10 u^{2}}{u^{2}+u_{H}^{2}}+\log \left(1+\frac{u^{2}}{u_{H}^{2}}\right)\right] \\
\hat{\mathcal{B}}_{2}(u)= & \frac{1}{24}\left[-\frac{u^{2}\left(11 u^{4}+3 u^{2} u_{H}^{2}+2 u_{H}^{4}\right)}{\left(u^{2}+u_{H}^{2}\right)^{2}}+2 u_{H}^{2} \log \left(1+\frac{u^{2}}{u_{H}^{2}}\right)\right], \\
\phi_{0}(u)= & 0, \\
\hat{\phi}_{0}(u)= & -\frac{u_{H}^{2}}{4} \log \left(1+\frac{u^{2}}{u_{H}^{2}}\right), \\
\hat{\phi}_{2}(u)= & \frac{1}{4}\left[-2 u^{2}+\frac{u^{4}}{u^{2}+u_{H}^{2}}+2 u_{H}^{2} \log \left(1+\frac{u^{2}}{u_{H}^{2}}\right)\right] .
\end{aligned}
$$

The gauge field takes the form,

$$
A_{t}=\frac{q}{8 \sqrt{3} u_{H}^{3}}\left[24\left(u_{H}^{2}-u^{2}\right)+5 a^{2} u_{H}^{2}\left(u^{2} \log \left(1+\frac{u^{2}}{u_{H}^{2}}\right)-u_{H}^{2} \log 2\right)\right]
$$




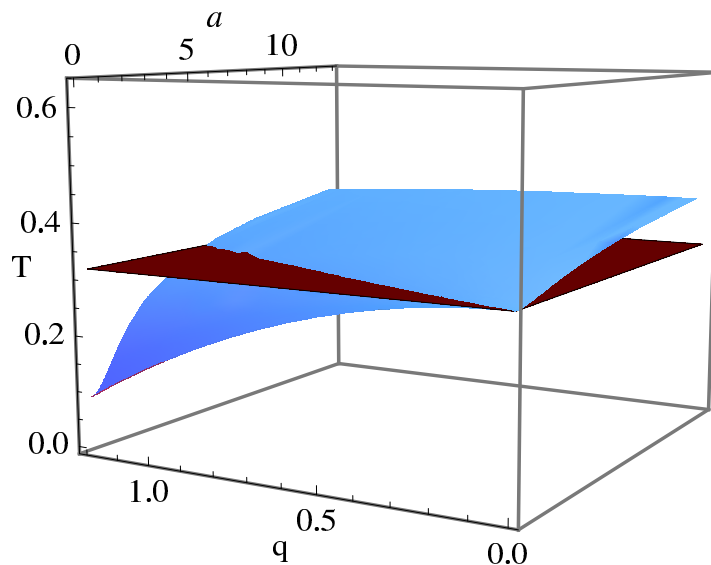

(a)

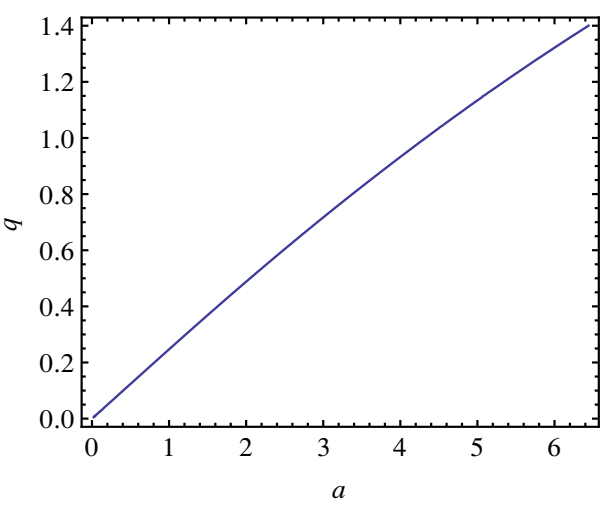

(b)

Figure 2. (a) shows the temperature of the AdS-BH (red surface) and that of the anisotropic, charged black brane (blue surface) with varying $q$ and $a$. (b) shows the line in the $a-q$ plane along which the temperature of the anisotropic, charged black brane equals the AdS-BH temperature.

with the corresponding chemical potential

$$
\mu=\frac{q\left(24-5 a^{2} u_{H}^{2} \log 2\right)}{8 \sqrt{3} u_{H}} .
$$

The temperature now reads,

$$
T=\frac{2-q^{2}}{2 \pi u_{H}}+a^{2} \frac{u_{H}}{96 \pi}\left[10 \log 2-4+5(3+\log 2) q^{2}\right]+\mathcal{O}\left(a^{4}\right) .
$$

Note that for a fixed $u_{H}$, for $a=0, T$ decreases with increasing $q$, whereas by setting $q=0$ one finds that $T$ increases with rise in $a$. An interesting feature of the charged, anisotropic black brane solution is that when both these parameters are switched on, there exists a regime in the parameter space spanned by $a$ and $q$ where the brane temperature is less than that of an isotropic, uncharged solution, i.e., AdS-black hole (AdS-BH). In the opposite regime, the temperature is greater than that of a AdS-BH. The two regimes are separated by a line in the $a-q$ plane along which the transition takes place and the temperature equals that of the AdS-BH geometry. This has been shown clearly in figure 2. For small $a$ and $q$ the functional form of the transition line is given by,

$$
q(a)=\frac{1}{4} \sqrt{\frac{10 \log 2-4}{3}} a+\frac{5}{384}(3+\log 2) \sqrt{\frac{10 \log 2-4}{3}} a^{3}+\mathcal{O}(a)^{5} .
$$

\section{Drag force}

In this section we evaluate the drag force felt by a heavy quark moving through a strongly coupled, anisotropic, charged plasma. The analysis of drag force on a moving quark was first done in the seminal works $[44,45]$. Since then the collisional energy loss has been computed in various other contexts using the gauge/string duality. Following the holographic 
prescription suggested in $[44,45]$ we consider a heavy quark of mass $m$ moving through the thermal bath with a velocity $\vec{v}$. A simple phenomenological model that encapsulates the dynamics of the quark is given by,

$$
\frac{d \vec{p}}{d t}=-\mu \vec{p}+\vec{F}
$$

where $\mu$ denotes the coefficient of drag and $\vec{F}$ is an externally applied force. $\vec{p} \equiv m \vec{v}$ is the quark momentum. The steady state condition is then defined by $\mu \vec{p}=\vec{F}$ when the quark moves with constant velocity. It is worth noting at this stage, that by virtue of the anisotropy of the medium, the drag coefficient $\mu$ is no longer a number but is now matrixvalued which we write as $\mu=\left\{\mu_{1}, \mu_{2}, \mu_{3}\right\}$. Moreover, the $\mathrm{SO}(2)$ invariance in the $x^{1}-x^{2}$ plane dictates that $\mu_{1}=\mu_{2}$. In the dual gravity picture a fundamental quark corresponds to a string embedded in the geometry (eq. (2.3)), hanging from the boundary at $u=0$ towards the horizon at $u=u_{H}$. The string dynamics is captured by the Nambu-Goto string world-sheet action,

$$
S_{\mathrm{NG}}=-\frac{1}{2 \pi \alpha^{\prime}} \int d \vec{\xi} \sqrt{-\operatorname{det} g_{\alpha \beta}} \equiv \int d \vec{\xi} \mathcal{L}
$$

where $g_{\alpha \beta}$ denotes the pull-back,

$$
g_{\alpha \beta}=G_{M N} \partial_{\alpha} X^{M} \partial_{\beta} X^{N}
$$

and $\vec{\xi} \equiv\left\{\xi^{0}, \xi^{1}\right\} \equiv\{\tau, \sigma\}$ are the coordinates defined on the string world-sheet. The space-time momentum flow is given by,

$$
\Pi_{M}=\frac{\partial \mathcal{L}}{\partial\left(\partial_{\sigma} X^{M}\right)} .
$$

As alluded to earlier, the quark is held in steady state by some external force. Physically this can be realized by turning on an electric field $F_{M N}=\partial_{[M} A_{N]}$ on the D7-brane worldvolume, to which the end-point of the fundamental probe string is attached. This implies that the action (eq. (3.2)) is now modified by the addition of a boundary term

$$
S=S_{\mathrm{NG}}+S_{\mathrm{b}}
$$

where

$$
S_{\mathrm{b}}=-\int_{\partial \Sigma} d \tau A_{M} \partial_{\tau} A^{M}=-\frac{1}{2} \int_{\partial \Sigma} d \tau F_{M N} X^{M} \partial_{\tau} X^{N}
$$

While the boundary term does not affect the dynamics of the string in the bulk, it modifies the boundary conditions that the string end-point has to satisfy. Setting $\delta S=0$ results in the boundary condition,

$$
\Pi_{M}+\left.F_{M N} \partial_{\tau} X^{N}\right|_{\partial \Sigma}=0
$$

To proceed further, we need to fix a convenient gauge. First note that the string does not move along the directions of $S^{5}$ so that its coordinates on $S^{5}$ are fixed. Further, rotational invariance in the $x^{1}-x^{2}$ plane allows us the freedom to set $x^{2}=0$. Now we 
employ the gauge-fixing as $\vec{\xi}=\{t, u\}$ and consider the string end-point on the boundary (or, equivalently, the quark) to be moving at an angle $\psi$ to the $x^{3}$ direction. We focus on string embedding of the form,

$$
\begin{aligned}
& X^{1}(t, u)=(v t+\rho(u)) \sin \psi, \\
& X^{3}(t, u)=(v t+\zeta(u)) \cos \psi
\end{aligned}
$$

The relevant boundary conditions are,

$$
\begin{aligned}
& X^{1}(t, 0) \equiv x^{1}(t)=v t \sin \psi, \\
& X^{3}(t, 0) \equiv x^{3}(t)=v t \cos \psi
\end{aligned}
$$

which is mapped to the trajectory of the quark on the boundary gauge theory. With this choice, the Lagrangian takes the explicit form, ${ }^{3}$

$$
\mathcal{L}=-\sqrt{\frac{\mathcal{B F}+\sin ^{2} \psi\left(\mathcal{B F} \mathcal{F}^{2} \rho^{\prime 2}-v^{2}\right)+\mathcal{H} \cos ^{2} \psi\left(\mathcal{B F}{ }^{2} \zeta^{\prime 2}-v^{2}-\mathcal{F} v^{2}\left(\rho^{\prime 2}-\zeta^{\prime 2}\right)^{2} \sin ^{2} \psi\right)}{\mathcal{F} u^{4}}} .
$$

It is now straight forward to compute the rate at which the energy and the momentum is transferred down the string towards the horizon,

$$
\begin{aligned}
-\Pi_{0} & =\frac{1}{\mathcal{L} u^{4}} \mathcal{B F} v\left[\rho^{\prime} \sin ^{2} \psi+\mathcal{H} \zeta^{\prime} \cos ^{2} \psi\right], \\
\Pi_{1} & =\frac{1}{\mathcal{L} u^{4}}\left[\mathcal{B F} \rho^{\prime}+\mathcal{H} v^{2}\left(\zeta^{\prime}-\rho^{\prime}\right) \cos ^{2} \psi\right] \sin \psi, \\
\Pi_{3} & =\frac{1}{\mathcal{L} u^{4}} \mathcal{H}\left[\mathcal{B F} \zeta^{\prime}+v^{2}\left(\rho^{\prime}-\zeta^{\prime}\right) \sin ^{2} \psi\right] \cos \psi .
\end{aligned}
$$

Correspondingly, the boundary conditions given in eq. (3.7) assume the form,

$$
\Pi_{1}=F_{1}, \quad \Pi_{3}=F_{3}, \quad-\Pi_{0}=F_{1} v \sin \psi+F_{3} v \cos \psi
$$

where $\vec{F}=\left\{F_{1}, F_{3}\right\}$ is the force needed to maintain the steady motion of the string. Intuitively, we expect the string to trail behind the quark which is ensured only when $\rho^{\prime}$ and $\zeta^{\prime}$ are negative. This, in turn, signifies that the direction of the energy and the momentum flow is from $u=0$ to $u=u_{H}$. It was argued in [23] that the string does not trail just below (along the radial direction) the quark - rather there is a misalignment between the transverse position of the quark and the trailing string and the same picture emerges here. To evaluate the string profile we rearrange eq. (3.11) to obtain,

$$
\begin{aligned}
\rho^{\prime} & = \pm \sqrt{\frac{\mathcal{H}}{\mathcal{B}}} \frac{v}{\mathcal{F}} \frac{P_{1}}{\sqrt{P_{1} P_{3}-N}}, \\
\zeta^{\prime} & = \pm \frac{v}{\mathcal{F} \sqrt{\mathcal{B H}}} \frac{P_{3}}{\sqrt{P_{1} P_{3}-N}}
\end{aligned}
$$

where we have introduced the following quantities,

$$
P_{1}=-\Pi_{1}\left[\mathcal{B F} \csc \psi-v^{2} \sin \psi\right]+\Pi_{3} v^{2} \cos \psi
$$

\footnotetext{
${ }^{3}$ We have suppressed the factor $1 / 2 \pi \alpha^{\prime}$ for the time being. We shall reinstate this factor at the end.
} 


$$
\begin{aligned}
P_{3} & =-\Pi_{3}\left[\mathcal{B F} \sec \psi-\mathcal{H} v^{2} \cos \psi\right]+\Pi_{1} \mathcal{H} v^{2} \sin \psi \\
N & =\frac{\mathcal{B} \mathcal{F} \csc \psi \sec \psi}{u^{4}}\left[\Pi_{1} \Pi_{3} u^{4}-\mathcal{H} v^{2} \cos \psi \sin \psi\right]\left[\mathcal{B F}-v^{2}\left(\mathcal{H} \cos ^{2} \psi+\sin ^{2} \psi\right)\right] .
\end{aligned}
$$

To evaluate the momenta we closely follow the argument developed in [23]. First note that the quantity $\left(P_{1} P_{3}-N\right)_{\left.\right|_{u=0, u_{H}}}>0$. However, generically, $P_{1} P_{3}-N$ can become negative in some range $u_{1}<\delta u<u_{2}$ where $0<u_{1}<u_{2}<u_{H}$ and $\left(P_{1} P_{3}-N\right)_{\left.\right|_{u=u_{1}, u_{2}}}=0$. Thus, within $\delta u$ both $\rho^{\prime}$ and $\zeta^{\prime}$ become imaginary-valued which is unacceptable. To circumvent this pathology, we demand that $P_{1} P_{3}-N \geq 0$ in the range $0 \leq u \leq u_{H}$. This implies that the two zeros of $P_{1} P_{3}-N$ at $u_{1}, u_{2}$ coincide. We denote the radial position of this zero by $u_{c}$. In such a scenario, $P_{1} P_{3}-N$ is always real and positive barring at $u=u_{c}$ where we demand that the numerators in eq. (3.13) also vanish. Then, both $\rho^{\prime}$ and $\zeta^{\prime}$ are smooth and negative in the range $0 \leq u \leq u_{H}$ provided that in eq. (3.13) the positive sign is chosen for $u<u_{c}$ and the negative sign is chosen for $u>u_{c}$. The simultaneous vanishing of the numerator and the denominator has the consequence that the zeros of $P_{1}, P_{3}$ and the two zeros of $N$ coincide which permits us to solve for the two momenta independently. First of all, setting the second term in $P_{1} P_{3}-N$ to be zero we find $u_{c}$

$$
\mathcal{B}_{c} \mathcal{F}_{c}-v^{2}\left(\mathcal{H}_{c} \cos ^{2} \psi+\sin ^{2} \psi\right)=0
$$

where $\mathcal{B}_{c} \equiv \mathcal{B}\left(u_{c}\right)$ and so on. Using eq. (3.15) in either $P_{1}$ or $P_{3}$ yields a relation between the two momenta

$$
\Pi_{1} \mathcal{H}_{c} \cos \psi=\Pi_{3} \sin \psi
$$

Finally, setting the first term in $P_{1} P_{3}-N$ to zero yields the exact expressions for the two momenta

$$
\Pi_{1}=\frac{v \sin \psi}{u_{c}^{2}}, \quad \Pi_{3}=\frac{\mathcal{H}_{c} v \cos \psi}{u_{c}^{2}} .
$$

Hence, the drag force $\vec{F}$ exerted by the medium upon the massive quark moving with constant velocity $\vec{v}=v\{\sin \psi, \cos \psi\}$ is given by ${ }^{4}$

$$
\vec{F}=\left\{F_{1}, F_{3}\right\}=\left\{\Pi_{1}, \Pi_{3}\right\}=\frac{\sqrt{\lambda}}{2 \pi} \frac{v}{u_{c}^{2}}\left\{\sin \psi, \mathcal{H}_{c} \cos \psi\right\}
$$

Also note that we have reinstated the factor $1 / 2 \pi \alpha^{\prime}=\sqrt{\lambda} / 2 \pi$ in our expression invoking, in the process, the gauge/string dictionary. Further, it is to be observed that the curious feature of the drag force not being aligned with the velocity as found in the anisotropic medium in [23], continues to hold here, as expected. In the simplifying limit $a \rightarrow 0$ and $q \rightarrow 0$ the expression above reduces to the drag force in $\mathcal{N}=4$ SYM plasma,

$$
\vec{F}_{a=0, q=0}=\left\{F_{1}, F_{3}\right\}=F_{0}\{\sin \psi, \cos \psi\}
$$

\footnotetext{
${ }^{4}$ Strictly speaking, the external force $\vec{F}$ is the negative of the drag force. But we shall be sloppy in our convention and refer to $\vec{F}$ itself to be the drag force.
} 


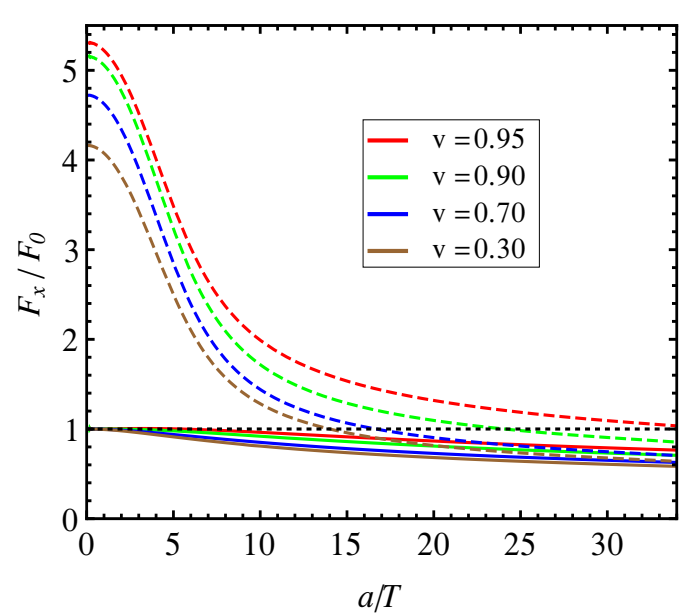

(a)

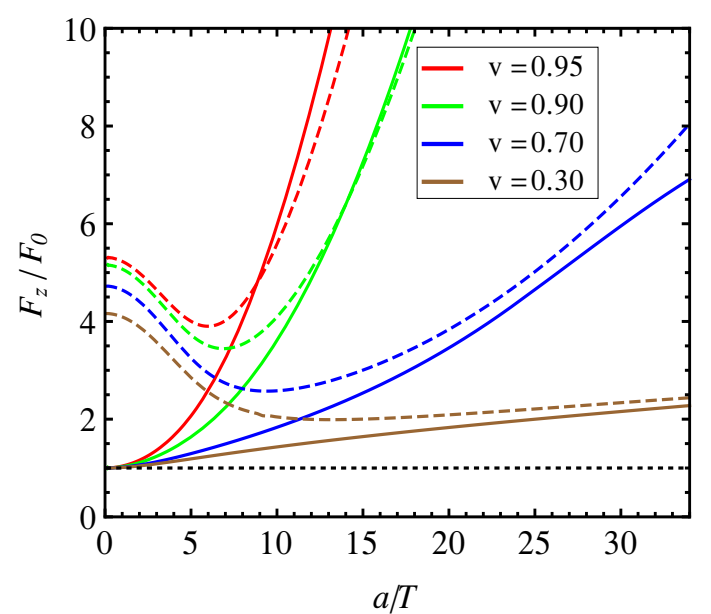

(b)

Figure 3. Variation of $F_{x}$ (a) and $F_{z}$ (b) with $a / T$ when $q=0,1$ at different values of $v=$ $0.30,0.70,0.90,0.95$.

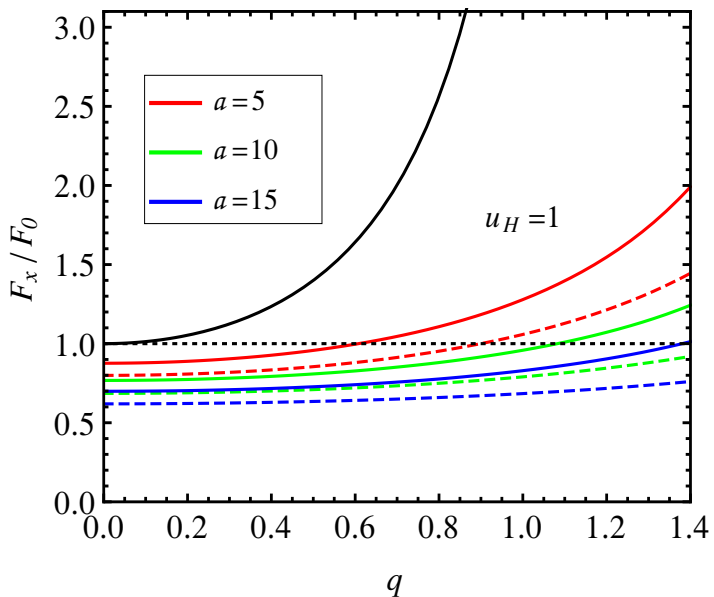

(a)

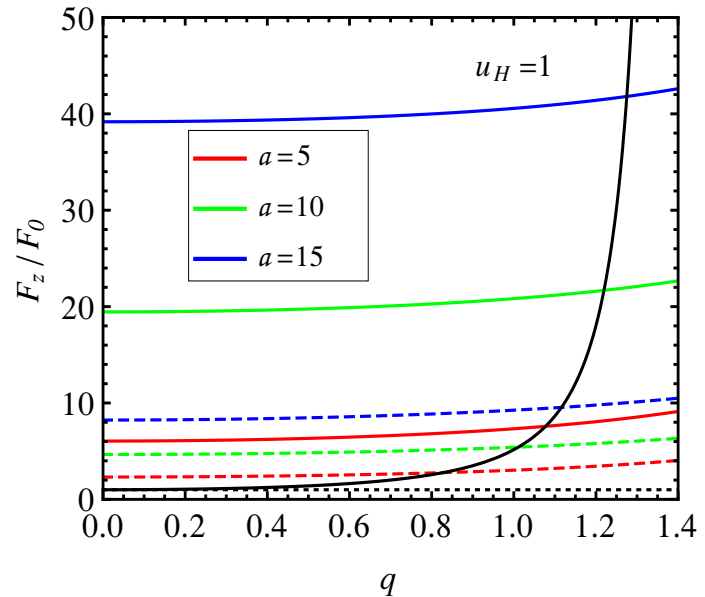

(b)

Figure 4. Variation of $F_{x}(\mathrm{a})$ and $F_{z}(\mathrm{~b})$ with $q$ when $a$ is fixed at $a=5,10,15$ and $v=0.7,0.9$.

where

$$
F_{0}=\frac{\pi}{2} \sqrt{\lambda} T^{2} \frac{v}{\sqrt{1-v^{2}}}
$$

and we have used,

$$
u_{c}^{2}=\frac{\sqrt{1-v^{2}}}{\pi^{2} T^{2}} .
$$

\subsection{Results}

Having developed the requisite formalism, we shall now provide the numerical results. First of all, note that the drag force $\vec{F}$ depends upon the temperature $T$, the velocity $\vec{v}$ of the 
massive quark, defined by its magnitude $v$ and direction, $\psi$ with respect to the anisotropic axis $x^{3}$, the strength of the anisotropy parameter $a$ and also the charge density $q$. Thus, $\vec{F}$ has a five parameter dependence, which we denote by $F(T, v, \psi, a, q)$. In particular, we shall use the notation $F_{x}=F(T, v, \pi / 2, a, q)$ and $F_{z}=F(T, v, 0, a, q)$.

In figure 3 we provide the variation of both $F_{x}$ (figure $3(\mathrm{a})$ ) and $F_{z}$ (figure $3(\mathrm{~b})$ ) with the anisotropy parameter $a$ (scaled by the temperature $T$ ) when the charge density $q$ and the velocity $v$ are held fixed. The continuous lines stand for $q=0$ whereas the dashed lines stand for $q=1$. Moreover, we have considered four different values of the velocity $v=0.30,0.70,0.90,0.95$ as is evident from the figure. Further, for convenience in comparison we have also scaled by the drag force in an isotropic, uncharged plasma, which is represented by the black, dotted line and which we denote by $F_{0}=F(T, v, a=0, q=0)$. Let us first concentrate on $F_{x}$. We find that when $q=0, F_{x}$ starts from $F_{0}$ and then gradually decreases with $a$ for all the values of the velocity considered. This is in perfect agreement with the results reported earlier in [23]. Once we turn on a non-zero value of $q, F_{x}$ starts from a much higher value at $a=0$, which is attributed to the presence of the charge. But then the effect of $a$ takes control, and $F_{x}$ diminishes steadily with increasing $a$. While the rate of decrease is intially steep, it becomes softer at large values of $a . F_{x}$ crosses $F_{0}$ at some critical value of $a=a_{0}$, which will, in general, depend upon both $q$ and $v$. We find that the smaller the velocity, the sooner will $F_{x}$ come below $F_{0}$. Moreover, at large values of $a$ the curves corresponding to $q=0$ and $q=1$ come close together (for each value of $v$ ) indicating that at large values of anisotropy, the effect of charge ceases to have much significance. Coming to $F_{z}$ one finds that in the absence of any charge, $q=0, F_{z}$ increases monotonically with $a$ for any value of $v$. While for small values of $v$ it increases mildly, for higher values of $v$, the increase is much severe. However, the moment one introduces a finite charge density $q$ into the system it leads to an intriguing effect. We observe that in a charged plasma $F_{z}(a=0)$ initially starts off with a much higher value compared to $F_{0}$, an effect attributed to $q$. But then for small values of $a, F_{z}$ starts to decrease until at a critical value of $a=a_{m}$ it attains a minima $F_{z(\min )} . F_{z(\min )}$ will depend upon the value of $v$ - for larger $v$, the minima is attained at a smaller $a_{m}$. However, the value of $F_{z(\min )}$ is smaller for lower values of $v$. Beyond $a_{m}, F_{z}$ continues to rise closely following the pattern of $F_{z}(q=0)$ - slowly for smaller $v$ and violently for larger $v$.

Let us next come to figure 4 which shows the variation of $F_{x}$ and $F_{z}$ with $q$ for different values of $v$ and $a$. Here we have considered three values of $a=5,10,15$. The continuous lines stand for $v=0.9$ whereas the dotted lines stand for $v=0.7$. Again we have scaled our results by $F_{0}$, denoted by the black dotted line, for convenience in comparison. Let us first concentrate on $F_{x}$. In the absence of anisotropy, $F_{x}$ starts from $F_{0}$ and from there on increases significantly with increasing $q$, which is shown by the black, continuous curve (corresponding to $v=0.9$ ). In the presence of anisotropy, $F_{x}$ still increases, but now the rate of increase is toned down, with the suppression being more for greater values of $a$. Further, now one can identify a critical value of $q=q_{0}$ such that for $q<q_{0}$, one has $F_{x}<F_{0}$, on the other hand, for $q>q_{0}$ one has $F_{x}>F_{0}$. This is expected since we found earlier that anisotropy pushes $F_{x}$ down below $F_{0}$. It is then only natural that this effect will be present in the presence of charge too, until, the effect of $q$ becomes strong enough 


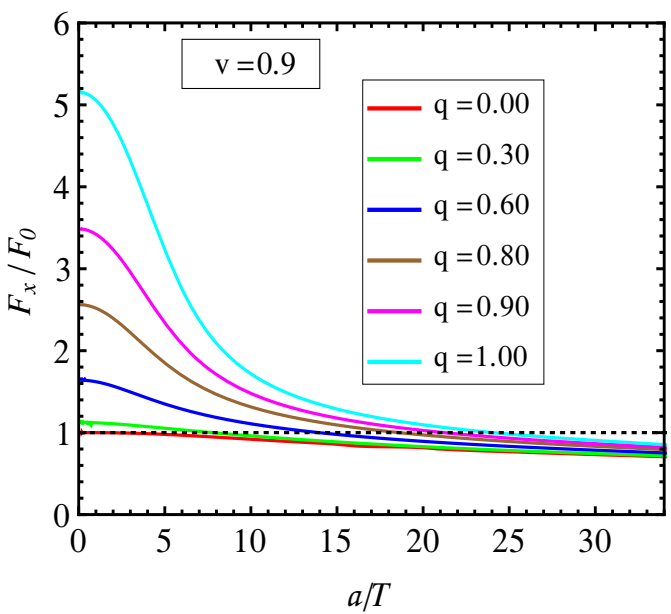

(a)

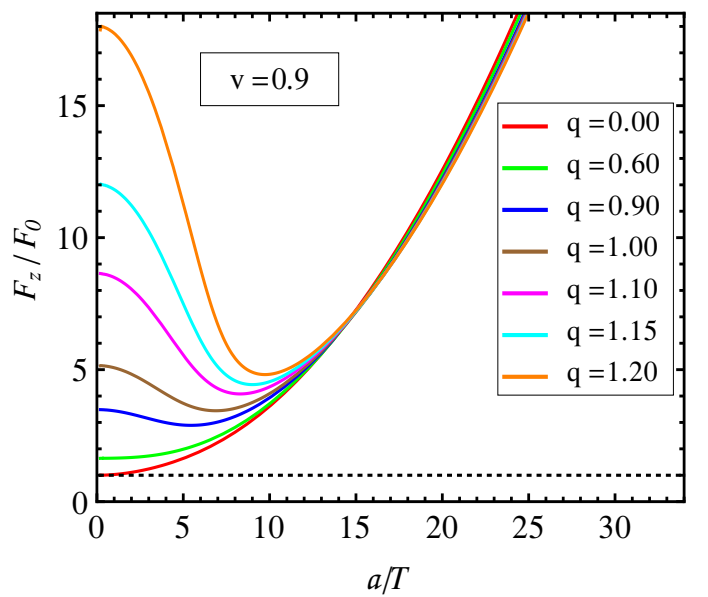

(b)

Figure 5. Variation of $F_{x}(\mathrm{a})$ and $F_{z}$ (b) with $a / T$ for fixed velocity $v=0.9$ and different values of $q$.

to again pull $F_{x}$ above $F_{0}$. The value of $q_{0}$ will depend both upon $a$ and $v$. It is found that $q_{0}$ increases with increasing $a$ and also with decreasing $v$. Next we move over to $F_{z}$ in figure 4(b). The black, continuous curve shows the variation with $q$ when $a=0$ and $v=0.9$. As one introduces anisotropy, $F_{z}$ increases compared to $F_{0}$ even at $q=0$ and for any velocity. Then it continues to rise with increase in $q$, but surprisingly, the rate of increase is now much subdued.

In figure 5 we again plot both $F_{x}$ and $F_{z}$ against the anisotropy parameter $a$ (scaled down by $T$ ), but now for a fixed $v=0.9$ and different values of the charge density $q$. As usual, the black curve stands for $F_{0}$. As one gradually increases $q, F_{x}(a=0)$ rises over $F_{0}$. But then for non-zero $a$, its effect starts to be felt. Consequently, $F_{x}$ decreases for all the values of $q$ considered, crosses $F_{0}$ at $a=a_{0}$ and then slowly tapers away. Again, the exact value of $a_{0}$ will depend upon $q$ - a smaller $q$ results in a smaller $a_{0}$. Also note that when $a$ is large, $F_{x}$ for different $q$ 's almost merge indicating that the effect of $a$ reigns over that of $q$. In $F_{z}$ also we find that with $q$ turned on, $F_{z}$ begins with a much higher value compared to $F_{0}$. In particular, for higher $q, F_{z}(a=0)$ will also be higher. Then we find a classic instance of the interplay between $a$ and $q$. For small values of $q \sim 0.6, F_{z}$ continues to rise while for larger values, e.g. $q \sim 0.9$ onwards, $F_{z}$ decreases to reach a minima $F_{z(\min )}$, beyond which it again rises. Of course, for different $q$, the minima $F_{z(\min )}$ is attained at different $a_{m}$. Ultimately, $F_{z}$ for all the values of $q$ almost merge at around $a \sim 15$ and then continues to rise implying that the effect of $q$ is insignificant at large $a$. Of course, we expect the value of $a$ where all the $F_{z}$ curves, corresponding to different $q$ 's, merge to be dependent upon the velocity $v$.

Figure 6 shows the variation of the drag force (scaled by $F_{0}$ ) with the velocity $v$ when the value of the anisotropy parameter $a / T$ and the charge density $q$ are kept fixed. We have kept the ratio $a / T$ fixed at 5 and considered a set of values of $q$. From figure $6(\mathrm{a})$ we find that initially when $q=0, F_{x}<F_{0}$. It then increases with $v$ to cross $F_{0}$ at some value of $v=v_{0}$ determined by the specific value of $a / T$. As one puts in a small value of 


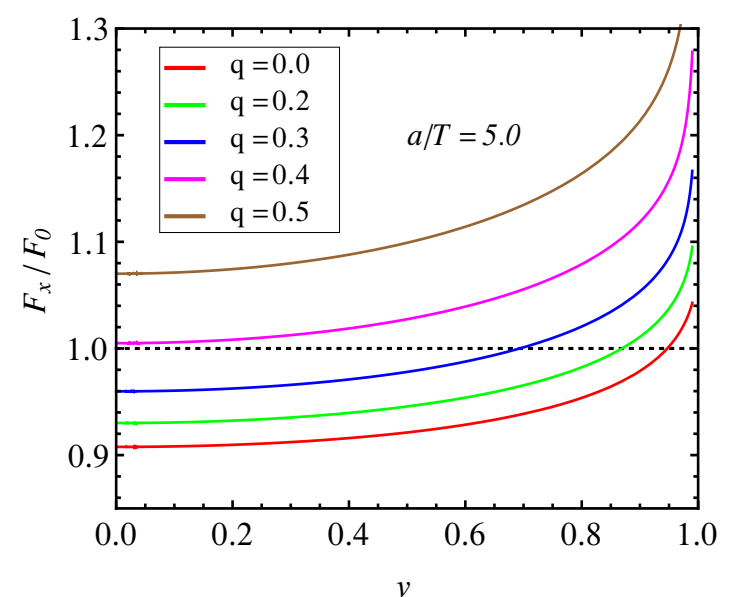

(a)

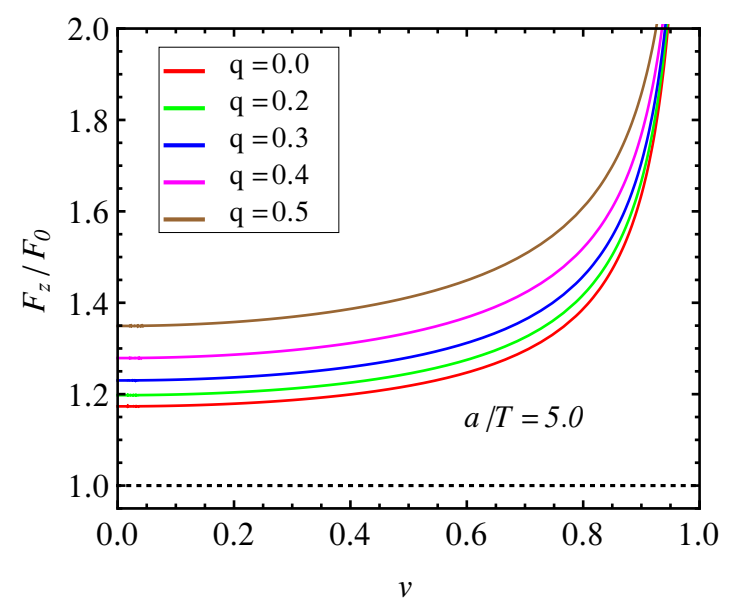

(b)

Figure 6. Variation of $F_{x}$ (a) and $F_{z}$ (b) with $v$ when $a / T=5$ and $q$ takes different fixed values.

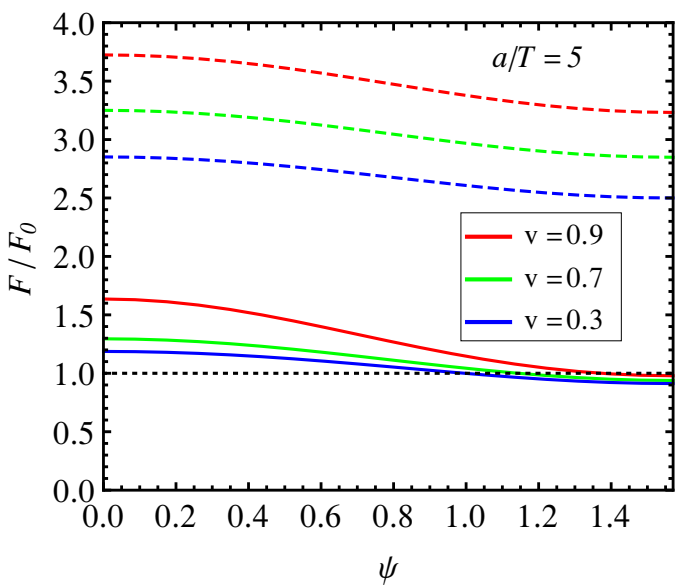

(a)

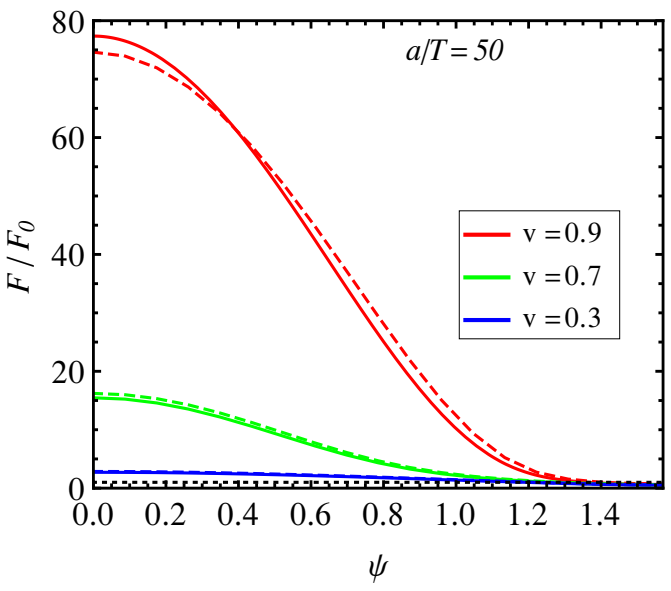

(b)

Figure 7. Variation of the drag force $F$ with the direction $\psi$ of the quark velocity (with respect to anisotropic direction) for different sets of $v$, at fixed $a$ and $q$.

the charge density $q$, the initial value of $F_{x}$ increases, though still remaining below $F_{0}$ but it now crosses $F_{0}$ at a smaller value of $v_{0}$. Finally, as $q$ is raised even further, $F_{x}$ becomes greater than $F_{0}$ to start with and then continually rises. The same pattern is noticed in the case of $F_{z}$ except that now $F_{x}>F_{0}$ for all the values of $q$ considered and throughout the range of $v$. Further, it is to be noticed that at large $v, F_{z}$ becomes practically independent of $q$. We shall also show this result analytically in the small anisotropy and small charge density limit to be discussed shortly.

Figure 7 shows how the drag force $F$ varies with the direction of the quark's velocity with respect to the anisotropy axis. We have considered two values of $a / T=5,50$ respectively in figure $7(\mathrm{a})$ and figure $7(\mathrm{~b})$. We have also considered $v=0.3,0.7,0.9$ and $q=0,1$ 


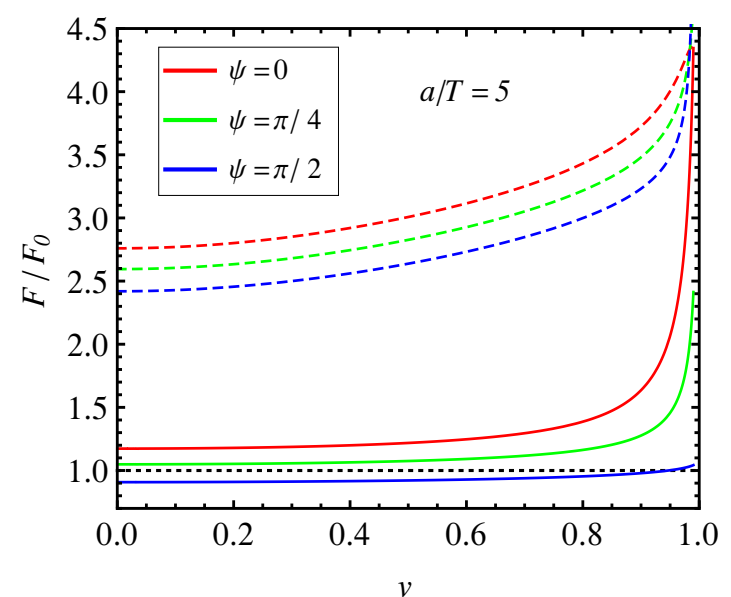

(a)

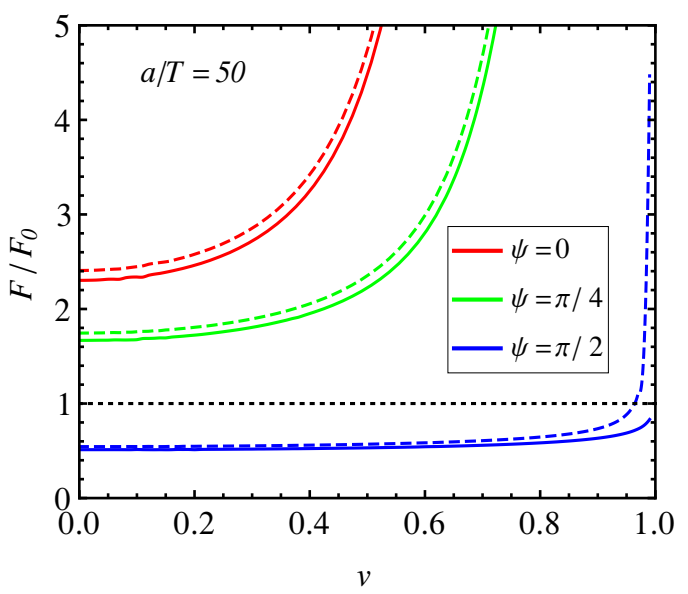

(b)

Figure 8. Variation of the drag force $F$ with $v$ for different orientations of the quark velocity and $q=0,1$.

represented by continuous and dashed curves respectively. The direction of anisotropy has $\psi=0$ while $\psi=\pi / 2$ represents the transverse plane. Note that, in general, the drag force along the anisotropic direction is greater than that in the transverse plane, irrespective of the values of $v, a$ and $q$. For moderate values of anisotropy, the drag force witnesses a small decrease with increasing $\psi$. But when the anisotropy parameter $a$ is large enough (as in figure $7(\mathrm{~b}))$ the slump in the drag force is more violent. Figure $7(\mathrm{a})$ shows that for moderate value of $a$ and $q=0$ the drag force is sensitive to the velocity for small $\psi$ whereas for large $\psi$ the drag force becomes effectively independent of $v$. On the other hand, for $q=1$, the velocity-dependence of the drag force persists throughout the allowed range of $\psi$ and the curves corresponding to different values of $v$ are equidistant from each other for any direction of the quark velocity. Another important observation is that as $\psi$ increases, for $q=1$ the drag force is always greater than $F_{0}$. But when the plasma is uncharged, the drag force becomes less than its isotropic, uncharged counterpart at a particular $\psi=\psi_{0}$. Thus, as the projection of the quark velocity in the transverse plane increases, at a certain $\psi_{0}$, that will, in general, depend upon $v$ and $a$, the drag force decreases below $F_{0}$. On the contrary, when the anisotropy is strong enough, one notes from figure 7 (b) that while the drag force is highly sensitive to the velocity for small $\psi$, the charge density has a meager effect on the drag force. This is evident from the fact that the curves for $q=0$ and $q=1$ merge with each other. Moreover, for large $\psi$, i.e., when the quark velocity has a significant component in the transverse plane, the drag force becomes effectively independent of the velocity and the charge density. In this case, the drag force becomes smaller than $F_{0}$ only for large $\psi$.

Figure 8 shows how the force varies with $v$ along different directions. We have considered three cases where $\psi=0, \pi / 4, \pi / 2$. We have also taken $q=0,1$, indicated respectively by continuous and dashed lines, to capture the effect of charge density. Further, we consider two values of $a / T$. First of all, observe from figure 8 (a) that in the uncharged case $q=0$ 
and moderate $a / T \sim 5$, the drag force increases mildly with $v$ for any direction of the quark velocity, up to a certain value of $v$, beyond which the rise in drag force becomes very steep. Also, when the motion is confined in the transverse plane, the drag force remains less than $F_{0}$ up to a certain value of $v$. When the plasma is endowed with a finite charge density $q$, the drag force is initially dependent upon the direction of the quark velocity, but as the ultra-relativistic regime $v \rightarrow 1$ is approached, $F$ becomes independent of $q$. Figure $8(\mathrm{~b})$ shows that for sufficiently large $a / T \sim 50, F$ increases - initially slowly and then sharply, with $v$. While the drag force in different directions are well resolved, one notes that the curves for $q=1$ almost mimic those for $q=0$, i.e., $F$ is largely insensitive to the effect of a finite charge density.

\subsection{Small anisotropy and small charge limit analysis}

In the preceding section we have provided numerical results for the drag force for arbitrary values of the anisotropy parameter $a$ and the charge density $q$. Here we consider only small values of $a$ and $q$ and furnish some analytical results. While restricting to small anisotropy is mandatory in order to proceed analytically, the small $q$ restriction is imposed only to make things simple. Having furnished the relevant functions earlier in the small $a$ and small $q$ regime let us proceed straight away to the results. The drag force along the $x^{1}$ direction, $F_{x}$ is given in this limit by,

$$
\begin{aligned}
F_{x}(T, v, a, q)=\frac{\pi}{2} & \sqrt{\lambda} T^{2} \frac{v}{\sqrt{1-v^{2}}}\left[1+\frac{q^{2}\left(-1+v^{2}+3 \sqrt{1-v^{2}}\right)}{2 \sqrt{1-v^{2}}}+\frac{a^{2}}{24 \pi^{2} T^{2}\left(1-v^{2}\right)} \times\right. \\
& \left.\times\left[1-v^{2}+\sqrt{1-v^{2}}+\left(4 v^{2}-5\right) \log \left(1+\sqrt{1-v^{2}}\right)\right]\right] \\
=F_{0} & {\left[1+\frac{q^{2}\left(-1+v^{2}+3 \sqrt{1-v^{2}}\right)}{2 \sqrt{1-v^{2}}}+\frac{a^{2}}{24 \pi^{2} T^{2}\left(1-v^{2}\right)}\right.} \\
& \left.\times\left[1-v^{2}+\sqrt{1-v^{2}}+\left(4 v^{2}-5\right) \log \left(1+\sqrt{1-v^{2}}\right)\right]\right]
\end{aligned}
$$

where we have ignored terms of the $\mathcal{O}(a)^{4}, \mathcal{O}(q)^{4}$ and $\mathcal{O}(a q)^{2}$ and higher orders. In the non-relativistic limit it simplifies further as,

$$
\begin{aligned}
F_{x}(T, v, a, q)= & F_{0}\left[1+q^{2}\left(1+\frac{v^{2}}{4}+\mathcal{O}(v)^{4}\right)\right. \\
& \left.+\frac{a^{2}}{24 \pi^{2} T^{2}}\left(2-5 \log 2+\frac{7-4 \log 2}{4} v^{2}+\mathcal{O}(v)^{4}\right)\right]
\end{aligned}
$$

In the opposite regime, when the velocity is very high, $v \sim 1$, one can write $\epsilon(v)=1-v^{2}$ and the drag force assumes the form,

$$
F_{x}(T, v, a, q)=F_{0}\left[1+\frac{q^{2}}{2}(3-\sqrt{\epsilon(v)})+\frac{a^{2}}{16 \pi^{2} T^{2}}\left(1-\frac{26}{9} \sqrt{\epsilon(v)}\right)\right] .
$$


As alluded to earlier, the effect of $a$ is to suppress $F_{x}$ compared to $F_{0}$ whereas the effect of $q$ is to increase $F_{x}$ above $F_{0}$, which leads to an interplay when both the parameters are turned on. This enables us to identify a regime of $a$ and $q$ for which $F_{x}<F_{0}$ whereas in the complementary domain of the $a-q$ space $F_{x}>F_{0}$. The two domains are demarcated by a line in the $a-q$ plane where $F_{x}=F_{0}$. In the small $a$, small $q$ limit, this transition line obeys the equation,

$$
q(a)=\frac{1}{2 \pi} \frac{\sqrt{1-v^{2}+\sqrt{1-v^{2}}+\left(4 v^{2}-5\right) \log \left(1+\sqrt{1-v^{2}}\right)}}{\sqrt{\left(-1+v^{2}\right)\left(9-3 \sqrt{1-v^{2}}\right)}} \frac{a}{T}
$$

Again, in the non-relativistic limit $v \ll 1$, it admits an expansion,

$$
q(a)=\frac{a}{\pi T}\left[\frac{\sqrt{5 \log 2-2}}{2 \sqrt{6}}-v^{2} \frac{5+\log 2}{16 \sqrt{6(5 \log 2-2)}}+\mathcal{O}(v)^{4}\right]
$$

which shows the transition line to be a straight line with a slope that depends upon $v$.

In a similar fashion one can obtain an expression for the drag force $F_{z}$ along the anisotropic direction in the small $a$ and small $q$ limit

$$
\begin{aligned}
F_{z}(T, v, a, q)=F_{0}[1 & +q^{2} \frac{4\left(1+\sqrt{1-v^{2}}\right)+v^{2}\left(\sqrt{1-v^{2}}-1\right)}{2\left(1+\sqrt{1-v^{2}}\right)^{2}} \\
& \left.+\frac{a^{2}}{T^{2}} \frac{1-v^{2}+\sqrt{1-v^{2}}+\left(1+v^{2}\right) \log \left(1+\sqrt{1-v^{2}}\right)}{24 \pi^{2}\left(1-v^{2}\right)}\right]+\mathcal{O}\left(a^{4}, q^{4}, a^{2} q^{2}\right) .
\end{aligned}
$$

As in the case of $F_{x}$, here too we can consider two simplifying limits. In the non-relativistic limit $v \ll 1$ the above expression reduces to,

$$
\begin{aligned}
F_{z}=F_{0} & {\left[1+q^{2}\left(1+\frac{v^{2}}{4}+\mathcal{O}(v)^{4}\right)\right.} \\
& \left.+\frac{a^{2}}{24 \pi^{2} T^{2}}\left(2+\log 2+v^{2}\left(\frac{1+8 \log 2}{4}\right)+\mathcal{O}(v)^{4}\right)\right] .
\end{aligned}
$$

In the ultra-relativistic limit $v \sim 1$, as before we introduce $\epsilon(v)=1-v^{2}$, whence the expression for $F_{z}$ simplifies to,

$$
F_{z}=F_{0}\left[1+\frac{q^{2}}{2}(3-\sqrt{\epsilon(v)})+\frac{a^{2}}{8 \pi^{2} T^{2}}\left(\frac{1}{\sqrt{\epsilon(v)}}-\frac{\sqrt{\epsilon(v)}}{9}\right)\right] .
$$

Observe that as $v \rightarrow 1, \epsilon \rightarrow 0$ and the ratio $F_{z} / F_{0}$ diverges as $(\sqrt{\epsilon})^{-1}$. This is in agreement with the results of [23] where the authors report that unless the quark moves in the transverse plane, the above-mentioned ratio diverges. Also observe that at large $v$ the term depending upon $a^{2}$ dominates over the one depending upon $q^{2}$ so that in the large velocity regime, the drag force becomes practically independent of the charge density. This corroborates our earlier conclusion based on numerical analysis. 


\section{Discussion and conclusion}

Finally, let us close with a summary of the results obtained. In this paper, we focused on the drag force on a massive quark moving through a strongly coupled hot, anisotropic plasma that carries a U(1) charge density. We employed holographic techniques to explore the combined effect of anisotropy and charge density upon the drag force. While the effects of anisotropy and finite charge density were well-known previously, in this paper we explore the interplay between these parameters and uncover some interesting features when both these effects act in conjunction. While the effect of charge is to increase the drag force, the effect of anisotropy is to sharply raise the drag force along the direction of anisotropy and suppress the same in the transverse plane, in general. When both the effects act in unison, we find that the drag force in the transverse plane can become greater than its isotropic, uncharged counterpart in some regime of the parameter space. A rather remarkable observation is that while increasing $a$ or $q$ cause the drag force to shoot up, in the combined presence of both these effects, the rate of increase of the drag force along the anisotropic direction is mellowed down drastically. We also find that for strong anisotropy and when the quark trajectory is confined in the transverse plane, the drag force (scaled by $F_{0}$ ) is practically independent of the velocity and the charge density. On the other hand, for large anisotropy, the drag force (scaled) along the anisotropic direction is independent of $q$ but is affected by the velocity. In the lack of much analytical control, most of our conclusions hinged upon numerical plots. However, in the limit of weak anisotropy and small charge density, the computations become more amenable to analytical treatment and we were able to furnish analytical expressions for the drag force both along the anisotropic direction and in the transverse plane. We further considered the non-relativistic and the ultra-relativistic limits whence the results simplified greatly. A curious feature to come up is that in the presence of both anisotropy and charge density there exists a domain of values of $a$ and $q$ in which the drag force in the transverse plane is smaller than the isotropic, uncharged one $F_{0}$, whereas in the complementary domain the drag force is greater than $F_{0}$. The two domains are separated by a transition line in the $a-q$ parameter space along which $F_{x}=F_{0}$. We were also successful in giving the functional dependence of $q$ upon $a$ along which this transition takes place.

\section{A Details of the numerical scheme used}

The numerical scheme we follow to find out the various functions is primarily based on the one used in [41] excepting certain minor differences which we elucidate below. The relevant equations of motion are given by [41]

$$
\begin{aligned}
\mathcal{F} & =\frac{e^{-\frac{1}{2} \phi}}{12\left(\phi^{\prime}+u \phi^{\prime \prime}\right)}\left(3 a^{2} e^{\frac{7}{2} \phi}\left(4 u+u^{2} \phi^{\prime}\right)+48 \phi^{\prime}-2 e^{\frac{5}{2} \phi} Q^{2} u^{6} \phi^{\prime}\right), \\
\frac{\mathcal{B}^{\prime}}{\mathcal{B}} & =\frac{1}{24+10 u \phi^{\prime}}\left(24 \phi^{\prime}-9 u \phi^{\prime 2}+20 u \phi^{\prime \prime}\right), \\
0 & =\frac{-48 \phi^{\prime 2}\left(32+7 u \phi^{\prime}\right)+768 \phi^{\prime \prime}+4 e^{\frac{5 \phi}{2}} Q^{2} u^{5}\left(-24 \phi^{\prime}+u^{2} \phi^{\prime 3}-8 u \phi^{\prime \prime}\right)}{48 \phi^{\prime}-2 e^{\frac{5 \phi}{2}} Q^{2} u^{6} \phi^{\prime}+3 a^{2} e^{\frac{7 \phi}{2}} u\left(4+u \phi^{\prime}\right)}
\end{aligned}
$$




$$
\begin{aligned}
& +\frac{1}{u\left(12+5 u \phi^{\prime}\right)\left(\phi^{\prime}+u \phi^{\prime \prime}\right)}\left[13 u^{3} \phi^{4}+u^{2} \phi^{\prime 3}\left(96+13 u^{2} \phi^{\prime \prime}\right)+8 u\left(-60 \phi^{\prime \prime}+11 u^{2} \phi^{\prime \prime 2}-12 u \phi^{\prime \prime \prime}\right)\right. \\
& \left.+2 u \phi^{\prime 2}\left(36+53 u^{2} \phi^{\prime \prime}-5 u^{3} \phi^{\prime \prime \prime}\right)+\phi^{\prime}\left(30 u^{4} \phi^{\prime \prime 2}-64 u^{3} \phi^{\prime \prime \prime}-288+32 u^{2} \phi^{\prime \prime}\right)\right] .
\end{aligned}
$$

After inflicting the transformation

$$
\tilde{\phi}=\phi+\frac{4}{7} \log a
$$

as done in $[21,41]$ the differential equation for $\tilde{\phi}$ looks like

$$
\begin{aligned}
0= & \frac{-48 \tilde{\phi}^{\prime 2}\left(32+7 u \tilde{\phi}^{\prime}\right)+768 \tilde{\phi}^{\prime \prime}+4 e^{\frac{5 \tilde{\phi}}{2}} a^{-\frac{10}{7}} Q^{2} u^{5}\left(-24 \tilde{\phi}^{\prime}+u^{2} \tilde{\phi}^{3}-8 u \tilde{\phi}^{\prime \prime}\right)}{48 \tilde{\phi}^{\prime}-2 e^{\frac{5 \tilde{\phi}}{2}} a^{-\frac{10}{7}} Q^{2} u^{6} \tilde{\phi}^{\prime}+3 e^{\frac{7 \tilde{\phi}}{2}} u\left(4+u \tilde{\phi}^{\prime}\right)} \\
& +\frac{1}{u\left(12+5 u \tilde{\phi}^{\prime}\right)\left(\tilde{\phi}^{\prime}+u \tilde{\phi}^{\prime \prime}\right)}\left[13 u^{3} \tilde{\phi}^{\prime 4}+u^{2} \tilde{\phi}^{\prime 3}\left(96+13 u^{2} \tilde{\phi}^{\prime \prime}\right)+8 u\left(-60 \tilde{\phi}^{\prime \prime}+11 u^{2} \tilde{\phi}^{\prime \prime 2}-12 u \tilde{\phi}^{\prime \prime \prime}\right)\right. \\
& \left.+u \tilde{\phi}^{\prime 2}\left(36+53 u^{2} \tilde{\phi}^{\prime \prime}-5 u^{3} \tilde{\phi}^{\prime \prime \prime}\right)+\tilde{\phi}^{\prime}\left(30 u^{4} \tilde{\phi}^{\prime \prime 2}-64 u^{3} \tilde{\phi}^{\prime \prime \prime}-288+32 u^{2} \tilde{\phi}^{\prime \prime}\right)\right] .
\end{aligned}
$$

If we further use

$$
\bar{Q}=a^{-\frac{5}{7}} Q
$$

as done in [41] we can solve the equation for $\tilde{\phi}$ in terms of $u, \tilde{\phi}_{H}, u_{H}, \bar{Q}$ using the boundary conditions at the horizon $u=u_{H}$ as explained in detail in [41]. But to extract the effect of the charge density $Q$ (or, equivalently $q$ ) it is convenient to use the parameter $Q$ itself without going into $\bar{Q}$. So we solved eq. (A.5) for $\tilde{\phi}$ to get

$$
\tilde{\phi}=\tilde{\phi}\left(u, u_{H}, \tilde{\phi}_{H}, Q, a\right) \text {. }
$$

Also from eq. (A.4) we have

$$
a=e^{\frac{7}{4} \tilde{\phi}\left(u=0, u_{H}, \tilde{\phi}_{H}, Q, a\right)}
$$

where we used the fact that $\phi$ vanishes on the boundary. As R.H.S. of eq. (A.8) is itself a function of $a$, we can solve it for $a$ numerically in terms of $u_{H}, \tilde{\phi}_{H}$ and which is independent of $Q$ as expected. In course of the numerical evaluation we have set $u_{H}=1$, which effectively means that we can use $\tilde{\phi}_{H}$ as an independent parameter in terms of which one can express $a$.

Open Access. This article is distributed under the terms of the Creative Commons Attribution License (CC-BY 4.0), which permits any use, distribution and reproduction in any medium, provided the original author(s) and source are credited.

\section{References}

[1] STAR collaboration, J. Adams et al., Experimental and theoretical challenges in the search for the quark gluon plasma: the STAR collaboration's critical assessment of the evidence from RHIC collisions, Nucl. Phys. A 757 (2005) 102 [nucl-ex/0501009] [INSPIRE]. 
[2] PHENIX collaboration, K. Adcox et al., Formation of dense partonic matter in relativistic nucleus-nucleus collisions at RHIC: experimental evaluation by the PHENIX collaboration, Nucl. Phys. A 757 (2005) 184 [nucl-ex/0410003] [InSPIRE].

[3] E. Shuryak, Why does the quark gluon plasma at RHIC behave as a nearly ideal fluid?, Prog. Part. Nucl. Phys. 53 (2004) 273 [hep-ph/0312227] [InSPIRE].

[4] E.V. Shuryak, What RHIC experiments and theory tell us about properties of quark-gluon plasma?, Nucl. Phys. A 750 (2005) 64 [hep-ph/0405066] [INSPIRE].

[5] J.M. Maldacena, The large-N limit of superconformal field theories and supergravity, Int. J. Theor. Phys. 38 (1999) 1113 [hep-th/9711200] [InSPIRE].

[6] E. Witten, Anti-de Sitter space and holography, Adv. Theor. Math. Phys. 2 (1998) 253 [hep-th/9802150] [INSPIRE].

[7] S.S. Gubser, I.R. Klebanov and A.M. Polyakov, Gauge theory correlators from noncritical string theory, Phys. Lett. B 428 (1998) 105 [hep-th/9802109] [INSPIRE].

[8] O. Aharony, S.S. Gubser, J.M. Maldacena, H. Ooguri and Y. Oz, Large-N field theories, string theory and gravity, Phys. Rept. 323 (2000) 183 [hep-th/9905111] [InSPIRE].

[9] M. Martinez and M. Strickland, Dissipative dynamics of highly anisotropic systems, Nucl. Phys. A 848 (2010) 183 [arXiv:1007.0889] [InSPIRE].

[10] R. Ryblewski and W. Florkowski, Non-boost-invariant motion of dissipative and highly anisotropic fluid, J. Phys. G 38 (2011) 015104 [arXiv:1007.4662] [INSPIRE].

[11] M. Martinez and M. Strickland, Non-boost-invariant anisotropic dynamics, Nucl. Phys. A 856 (2011) 68 [arXiv: 1011.3056] [InSPIRE].

[12] R. Ryblewski and W. Florkowski, Highly-anisotropic and strongly-dissipative hydrodynamics with transverse expansion, Eur. Phys. J. C 71 (2011) 1761 [arXiv:1103.1260] [INSPIRE].

[13] J.M. Maldacena and J.G. Russo, Large-N limit of noncommutative gauge theories, JHEP 09 (1999) 025 [hep-th/9908134] [INSPIRE].

[14] M. Alishahiha, Y. Oz and M.M. Sheikh-Jabbari, Supergravity and large-N noncommutative field theories, JHEP 11 (1999) 007 [hep-th/9909215] [INSPIRE].

[15] T. Harmark and N.A. Obers, Phase structure of noncommutative field theories and spinning brane bound states, JHEP 03 (2000) 024 [hep-th/9911169] [INSPIRE].

[16] R.-G. Cai and N. Ohta, On the thermodynamics of large-N noncommutative super Yang-Mills theory, Phys. Rev. D 61 (2000) 124012 [hep-th/9910092] [INSPIRE].

[17] R.-G. Cai and N. Ohta, Noncommutative and ordinary super Yang-Mills on $(D(p-2), D p)$ bound states, JHEP 03 (2000) 009 [hep-th/0001213] [INSPIRE].

[18] S. Chakraborty and S. Roy, Calculating the jet quenching parameter in the plasma of NCYM theory from gauge/gravity duality, Phys. Rev. D 85 (2012) 046006 [arXiv:1105.3384] [INSPIRE].

[19] S. Chakraborty, N. Haque and S. Roy, Wilson loops in noncommutative Yang-Mills theory using gauge/gravity duality, Nucl. Phys. B 862 (2012) 650 [arXiv:1201.0129] [INSPIRE].

[20] D. Mateos and D. Trancanelli, The anisotropic $N=4$ super Yang-Mills plasma and its instabilities, Phys. Rev. Lett. 107 (2011) 101601 [arXiv:1105.3472] [INSPIRE]. 
[21] D. Mateos and D. Trancanelli, Thermodynamics and instabilities of a strongly coupled anisotropic plasma, JHEP 07 (2011) 054 [arXiv: 1106.1637] [INSPIRE].

[22] A. Rebhan and D. Steineder, Violation of the holographic viscosity bound in a strongly coupled anisotropic plasma, Phys. Rev. Lett. 108 (2012) 021601 [arXiv:1110.6825] [INSPIRE].

[23] M. Chernicoff, D. Fernandez, D. Mateos and D. Trancanelli, Drag force in a strongly coupled anisotropic plasma, JHEP 08 (2012) 100 [arXiv: 1202.3696] [INSPIRE].

[24] D. Giataganas, Probing strongly coupled anisotropic plasma, JHEP 07 (2012) 031 [arXiv: 1202.4436] [INSPIRE].

[25] M. Chernicoff, D. Fernandez, D. Mateos and D. Trancanelli, Jet quenching in a strongly coupled anisotropic plasma, JHEP 08 (2012) 041 [arXiv:1203.0561] [INSPIRE].

[26] A. Rebhan and D. Steineder, Probing two holographic models of strongly coupled anisotropic plasma, JHEP 08 (2012) 020 [arXiv:1205.4684] [INSPIRE].

[27] K.B. Fadafan and H. Soltanpanahi, Energy loss in a strongly coupled anisotropic plasma, JHEP 10 (2012) 085 [arXiv: 1206. 2271] [INSPIRE].

[28] M. Chernicoff, D. Fernandez, D. Mateos and D. Trancanelli, Quarkonium dissociation by anisotropy, JHEP 01 (2013) 170 [arXiv: 1208.2672] [INSPIRE].

[29] B. Müller and D.-L. Yang, Light probes in a strongly coupled anisotropic plasma, Phys. Rev. D 87 (2013) 046004 [arXiv: 1210.2095] [InSPIRE].

[30] L. Patino and D. Trancanelli, Thermal photon production in a strongly coupled anisotropic plasma, JHEP 02 (2013) 154 [arXiv:1211.2199] [INSPIRE].

[31] S. Chakraborty and N. Haque, Holographic quark-antiquark potential in hot, anisotropic Yang-Mills plasma, Nucl. Phys. B 874 (2013) 821 [arXiv:1212.2769] [InSPIRE].

[32] S.-Y. Wu and D.-L. Yang, Holographic photon production with magnetic field in anisotropic plasmas, JHEP 08 (2013) 032 [arXiv: 1305.5509] [INSPIRE].

[33] S.I. Finazzo and J. Noronha, Estimates for the thermal width of heavy quarkonia in strongly coupled plasmas from holography, JHEP 11 (2013) 042 [arXiv:1306.2613] [INSPIRE].

[34] K.B. Fadafan, D. Giataganas and H. Soltanpanahi, The imaginary part of the static potential in strongly coupled anisotropic plasma, JHEP 11 (2013) 107 [arXiv:1306.2929] [INSPIRE].

[35] S. Chakrabortty, S. Chakraborty and N. Haque, Brownian motion in strongly coupled, anisotropic Yang-Mills plasma: a holographic approach, Phys. Rev. D 89 (2014) 066013 [arXiv:1311.5023] [INSPIRE].

[36] V. Jahnke, A. Luna, L. Patiño and D. Trancanelli, More on thermal probes of a strongly coupled anisotropic plasma, JHEP 01 (2014) 149 [arXiv:1311.5513] [INSPIRE].

[37] D. Giataganas and H. Soltanpanahi, Heavy quark diffusion in strongly coupled anisotropic plasmas, JHEP 06 (2014) 047 [arXiv: 1312.7474] [INSPIRE].

[38] M. Ali-Akbari and D. Allahbakhshi, Meson life time in the anisotropic quark-gluon plasma, JHEP 06 (2014) 115 [arXiv: 1404.5790] [INSPIRE].

[39] M. Ali-Akbari and S.F. Taghavi, Chiral magnetic effect in the anisotropic quark-gluon plasma, arXiv: 1408.6361 [INSPIRE]. 
[40] L. Cheng, X.-H. Ge and S.-J. Sin, Anisotropic plasma with a chemical potential and scheme-independent instabilities, Phys. Lett. B 734 (2014) 116 [arXiv:1404.1994] [INSPIRE].

[41] L. Cheng, X.-H. Ge and S.-J. Sin, Anisotropic plasma at finite U(1) chemical potential, JHEP 07 (2014) 083 [arXiv: 1404.5027] [INSPIRE].

[42] E. Caceres and A. Guijosa, Drag force in charged $N=4$ SYM plasma, JHEP 11 (2006) 077 [hep-th/0605235] [INSPIRE].

[43] K.B. Fadafan, Charge effect and finite 't Hooft coupling correction on drag force and jet quenching parameter, Eur. Phys. J. C 68 (2010) 505 [arXiv:0809.1336] [INSPIRE].

[44] C.P. Herzog, A. Karch, P. Kovtun, C. Kozcaz and L.G. Yaffe, Energy loss of a heavy quark moving through $N=4$ supersymmetric Yang-Mills plasma, JHEP 07 (2006) 013 [hep-th/0605158] [INSPIRE].

[45] S.S. Gubser, Drag force in AdS/CFT, Phys. Rev. D 74 (2006) 126005 [hep-th/0605182] [INSPIRE]. 